\title{
Galaxy-scale Astrometry and Galactic rotation measurements with VERA
}

\section{Mareki Honma*}

Mizusawa VERA Observatory, NAOJ, Mitaka, Tokyo 181-8588

E-mail: mareki.honma@nao.ac.jp

We present recent results of Galaxy-scale astrometry with VERA (VLBI Exploration of Radio Astrometry). VERA has been conducting regular monitoring of Galactic radio sources since 2004, and we have already detected parallaxes for several sources, ranging from a few $100 \mathrm{pc}$ to 5 kpc. In this paper, we summarize the recent results of astrometric measurements and discuss the Galaxy rotation obtained with VERA.

The 9th European VLBI Network Symposium on The role of VLBI in the Golden Age for Radio Astronomy and EVN Users Meeting

September 23-26, 2008

Bologna, Italy

${ }^{*}$ Speaker. 


\section{Introduction}

VERA (VLBI Exploration of Radio Astrometry) is a new VLBI array dedicated to phase referencing VLBI astrometry (Honma, Kawaguchi \& Sasao 2000; Kobayashi et al. 2003). The array consists of four 20-m radio telescopes spread over Japan, with the maximum baseline length of $2300 \mathrm{~km}$. VERA's $20 \mathrm{~m}$ telescopes are installed with a dual beam system, with which one can simultaneously observe a reference and a target source to cancel out the phase fluctuation caused by tropospheric variation. The array construction has been finished in 2002, and since 2004 we have been carrying out astrometric observations of Galactic radio sources.

\section{Galactic Rotation Measurements}

Sgr A* is an important source for Galaxy-scale astrometry to derive Galactic constants such as $R_{0}$ and $\Theta_{0}$. VERA has been conducting an astrometric program on Sgr A* using the QSO J1745283 as position reference. Although the parallax has not yet been detected due to large calibration errors at low elevation, the proper motion of $\mathrm{Sgr} \mathrm{A}^{*}$ has been successfully measured. The position and the motion of Sgr A* with respect to J1745-283 is consistent with VLBA observations of Sgr $A^{*}$ (Reid and Brunthaler 2004), though our measurement accuracy is not as high as that of VLBA, mainly due to the difference of the monitoring period length (2 years for VERA, while 8 years for VLBA). When VERA's measurements in near future are combined with the VLBA results, it will hopefully improve the accuracy of Sgr A* proper motion, and hence the accuracy of $\Omega_{0} \equiv \Theta_{0} / R_{0}$.

W49N is another important source for Galactic rotation measurement as it is located nearly on the solar circle, with an estimated distance of $\sim 11 \mathrm{kpc}$. With VERA we have already detected its proper motion along the Galactic plane. The preliminary results of proper motion ( $6.5 \mathrm{mas} / \mathrm{yr}$ ), combined with the LSR velocity of the molecular clouds, indicate that the Galactic angular velocity is $\Omega_{0}=30.0+/-0.6 \mathrm{~km} / \mathrm{s} / \mathrm{kpc}$. This is consistent with the value obtained from Sgr A* $(29.45+/-$ $0.1 \mathrm{~km} / \mathrm{s} / \mathrm{kpc}$, Reid and Brunthaler 2004), indicating that Sgr A* has no systematic motion along the Galactic plane and hence is at rest at the center of the Galaxy. Such measurements of $\Omega_{0}$ independent of Sgr A* is important for constraining the peculiar motion of Sgr A* itself, and thus will provide better understanding on the nature of Sgr A* (e.g., excluding binary black hole system at the Galaxy's center).

For the outer Galaxy rotation, the parallax of $\mathrm{S} 269 \mathrm{H}_{2} \mathrm{O}$ masers $(\pi=189 \pm 8 \mu$ as, or $\mathrm{D}=5.28 \pm$ $0.24 \mathrm{kpc}$ ) as well as their proper motions were used to constrain the Galactic rotation velocity at the position of S269 (located at $\sim 13 \mathrm{kpc}$ from the Galaxy center). Honma et al.(2007) demonstrated that the rotation curve of the Galaxy is basically flat out to $\sim 13 \mathrm{kpc}$ based on the distance and proper motions of S269.

\section{Toward a Face-on View of the Galaxy}

So far we have measured parallaxes and proper motions of several Galactic radio sources, including some interesting sources such as Orion-KL (Hirota et al. 2007; Kim et al. 2008), NGC 281 (Sato et al. 2008), VY CMa (Choi et al. 2008) and so on. Figure 1 summarizes the current status of astrometric results from VERA, showing sources for which parallaxes and/or proper motions 


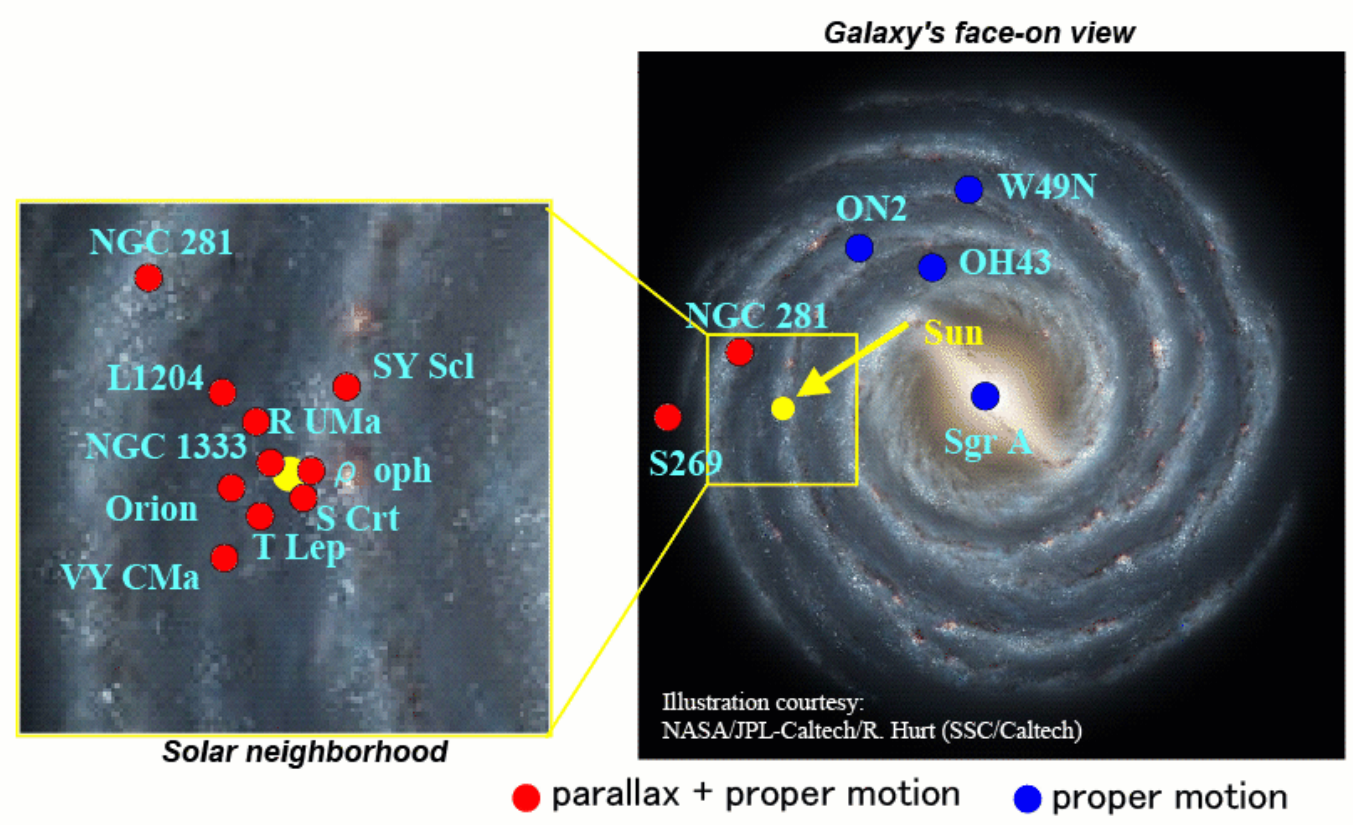

Figure 1: A schematic face-on view of the Milky Way Galaxy with locations of radio sources for which astrometric observations have been made with VERA. Red circles are those for which parallaxes and proper motions have been measured, and blue circles are those only with proper motion measurements.

have been measured. In addition to those sources shown in figure 1, totally $\sim 70$ sources have been monitored by summer 2008, and their data are to be analyzed for astrometric measurements. In 2008/2009 we will increase the number of observing sources up to $\sim 70$ per year, and in next $\sim 15$ years, VERA will observe totally $\sim 1000$ maser sources in the Galaxy, and precisely locate them in the Galaxy's disk to reveal the face-on view of the Galaxy.

\section{References}

[1] Choi, Y. K., et al. 2008, PASJ, 60, 1007

[2] Hirota, T., et al. 2007, PASJ, 59, 897

[3] Honma, M., Kawaguchi, N., Sasao, 2000, Proc. of SPIE Vol. 4015, 624

[4] Honma, M., et al. 2007, PASJ, 59, 889

[5] Kim, M. K., et al. 2008, PASJ, 60, 991

[6] Kobayashi, H., et al. 2003, in ASP Conf. Ser. 306, New Technologies in VLBI, ed. Y. C. Minh, 367

[7] Reid, M., Brunthaler, A., 2004, ApJ, 616, 872

[8] Sato, M., et al. 2008, PASJ, 60, 975 